\title{
Plasma D-dimer Can Effectively Predict the Prospective Occurrence of Ascites in Advanced Schistosomiasis Japonica Patients
}

\author{
Xiaoying $\mathrm{Wu}^{1}$, Jianwei Ren², Zulu Gao ${ }^{3}$, Yun $\mathrm{Xu}^{3}$, Huiqun $\mathrm{Xie}^{3}$, Tingfang $\mathrm{Li}^{4}$, Yanhua Cheng ${ }^{3}$, Fei Hư ${ }^{3}$, Hongyun $\mathrm{Liu}^{3}$, \\ Zhihong Gong ${ }^{3}$, Jinyi Liang ${ }^{5,6}$, Jia Shen ${ }^{5,6}$, Zhen Liu ${ }^{5,6}$, Feng Wu ${ }^{5,6}$, Xi Sun ${ }^{5,6}$, Zhongzheng Niu, \\ ${ }^{1}$ School of Public Health, Fudan University, Shanghai, China; ${ }^{2}$ Health Department of Guard Bureau of General Office of the Communist Party of \\ China, Beijing, P. R. China; ${ }^{3}$ Jiangxi Provincial Institute of Parasitic Diseases, Nanchang, P. R. China; ${ }^{4}$ Schistosomiasis Control Station of Yugan \\ County, Shangrao, P. R. China; ${ }^{5}$ Key Laboratory of Tropical Diseases and Control of the Ministry of Education, Guangzhou, P. R. China; ${ }^{6}$ Department \\ of Parasitology, Zhongshan School of Medicine, Sun Yat-Sen University, Guangzhou, P. R. China; ${ }^{7}$ School of Community and Global Health, \\ Claremont Graduate University, Claremont, California, USA
}

\begin{abstract}
China still has more than 30,000 patients of advanced schistosomiasis while new cases being reported consistently. D-dimer is a fibrin degradation product. As ascites being the dominating symptom in advanced schistosomiasis, the present study aimed to explore a prediction model of ascites with D-dimer and other clinical easy-achievable indicators. A case-control study nested in a prospective cohort was conducted in schistosomiasis-endemic area of southern China. A total of 291 patients of advanced schistosomiasis were first investigated in 2013 and further followed in 2014. Information on clinical history, physical examination, and abdominal ultrasonography, including the symptom of ascites was repeatedly collected. Result showed 44 patients having ascites. Most of the patients' ascites were confined in the kidney area with median area of $20 \mathrm{~mm}^{2}$. The level of plasma D-dimer and pertinent liver function indicators were measured at the initial investigation in 2013. Compared with those without ascites, cases with ascites had significantly higher levels of D-dimer $(0.71 \pm 2.44 \mu \mathrm{g} / \mathrm{L}$ vs $0.48 \pm 2.12 \mu \mathrm{g} / \mathrm{L}, P=0.005)$, as well ALB (44.5 vs 46.2 , g/L) and Type IV collagen (50.04 vs $44.50 \mu \mathrm{g} / \mathrm{L}$ ). Receiver operating characteristic curve analyses indicated a moderate predictive value of $\mathrm{D}$-dimer by its own area under curve (AUC) of $0.64(95 \% \mathrm{Cl}: 0.54-0.73)$ and the cutoff value as $0.81 \mu \mathrm{g} / \mathrm{L}$. Dichotomized by the cutoff level, D-dimer along with other categorical variables generated a prediction model with AUC of 0.76 (95\% Cl: $0.68-$ 0.89 ). Risks of patients with specific characteristics in the prediction model were summarized. Our study suggests that the plasma D-dimer level is a reliable predictor for incident ascites in advanced schistosomiasis japonica patients.
\end{abstract}

Key words: Schistosoma japonicum, D-dimer, ascites, prediction, schistosomiasis japonica, China

\section{INTRODUCTION}

Schistosomiasis japonica, caused by the infection of Schistosoma japonicum, is a widely distributed parasitic zoonoses that threatens human's health and social economic development $[1,2]$. The epidemic situation of the disease used to be serious in China. Comprehensive schistosomiasis control strategies in the past decades decreased a concrete number of infected cases from 1.52 million in 1989 to 0.12 million in 2013, and most of the endemic areas are controlled within the status of transmission interruption [2]. However, new challenges emerged

- Received 19 September 2016, revised 13 February 2017, accepted 14 February 2017.

*Corresponding authors (07046262@163.com; zhongzheng.niu@cgu.edu)

(c) 2017, Korean Society for Parasitology and Tropical Medicine

This is an Open Access article distributed under the terms of the Creative Commons Attribution Non-Commercial License (http://creativecommons.org/licenses/by-nc/4.0) which permits unrestricted non-commercial use, distribution, and reproduction in any

medium, provided the original work is properly cited. that the remaining disease transmission in the geographically complex areas turns to be more challengable and that more infected patients who were not treated thoroughly are now developing into the late stage, i.e., advanced schistosomiasis [3-5]. Meanwhile, a few patients who received the standard clinical treatment timely still keep on the development to advanced schistosomiasis [3,5-8]. According to a 2014 national wide report, 115,614 cases of $S$. japonicum infection were estimated, and 30,880 patients are suffering from advanced schistosomiasis. Advanced schistosomiasis is characterized by hepatosplenic conditions, such as periportal liver fibrosis, spleen enlargement and congestion, portal hypertension, and other serious sequelae. The late stage schistosomiasis is hitherto difficult, if not impossible, to be fully recovered. Thus, with an increasing proportion of advanced cases in the overall disease spectrum, researchers should provide effective prevention strategies with early detection, early diagnosis, and early treatment. 
Ascites is a common symptom in the late stage of schistosomiasis, which is caused by venous obstruction and portal hypertension with accumulation of granulomatous inflammation and fibrosis $[3,9]$. More frequency of ascites in the advanced schistosomiasis patients were reports from different endemic areas with prevalence of ascites as $60-90 \%$ in advanced cases $[10,11]$. In advanced schistosomiasis cases, the interaction of reinfection and failure in fibrosis treatment destroys the patient's liver and portal vein system, causing the symptoms of ascites, and eventually deplete patient's health and quality of life [12]. Therefore, it is important to pre-diagnose and provide prevention and treatment to those advanced patients who have great risk of ascites. However, few studies have done this research.

The pathophysiological facts of vascular injury and fibrosis underlying the development of advanced schistosomiasis may be capable to predict the risk of ascites occurrence. D-dimer, one of the degradation products of cross-link fibrin, has been validated as an accurate marker of vascular injury and fibrinolytic activity [13-15]. Studies have confirmed the potential sensitivity and specificity of D-dimer in early diagnosis of pulmonary embolism, lung cancer, and esophageal varix bleeding [16-18]. It was found regarding the mechanisms of advanced schistosomiasis that peripheral portal vasculature destructions in liver fibrosis increases the intrahepatic portal stress and causes ascites [19]. Thus, it is logical and plausible to employ D-dimer as a sensitive marker in the predication of ascites. In the present study, we aimed to explore the predictive value of D-dimer in the incident ascites in patients of advanced schistosomiasis during 1-year follow up.

\section{MATERIALS AND METHODS}

\section{Participants}

A case-control study nested in a prospective cohort was conducted within 4 fields of Xinjian, Nanchang, Duchang, and Yugan Counties in Jiangxi Province, P. R. China, all of which are serious endemic areas of schistosomiasis japonica. Using stratified multistage sampling method, a total of 291 advanced schistosomiasis patients, diagnosed by the criteria made by Ministry of Health of the People's Republic of China [20], were first investigated in March through May 2013. To minimize the confounding effects of other liver diseases, patients with hepatic cirrhosis or steatosis, or active consumption of alcohol were excluded. Advanced patients were treated with standard clinic practices which included basic treatment, symptomatic treatment, and etiology treatment in the following year. All patients were further followed up in June through September 2014 for clinical symptoms and abdominal ultrasound test. In the subsequent design of case-control study, 44 patients with ascites in year 2014, diagnosed by abdominal ultrasonography [21], were compared as cases with the other 247 patients without ascites as controls. This study was approved by the Human Research Ethics Committee of the Sun Yat-Sen University (no. 2014032) in China. All patients received an explanation about the scope of the study, such as objectives, procedures, and potential risks, and signed an informed consent statement before inclusion in the study.

\section{Data collection}

Clinical history query, physical examination, and abdominal ultrasonography were conducted in the interviews of 2013 and 2014. Information on demographics, history of schistosomiasis, physical features, hepatic fibrosis, portal internal diameter, and ascites was accessed. Additionally, in the initial investigation in 2013, venous blood samples were drawn from all of the investigated individuals under aseptic conditions without stasis using vacuum tubes (Lucky Nation Medical, China) containing $0.562 \mathrm{M}$ EDTA-K3. Sera were obtained after rapid centrifugation for $10 \mathrm{~min}$ at $2,000 \mathrm{~g}$, stored in $0.5 \mathrm{ml}$ aliquots at $-80^{\circ} \mathrm{C}$. Further, a set of biomedical tests measured the serum levels of SEA, value of D-dimer, albumin (ALB), globulin (GLB), thrombin time (TT), and coagulation factor VIII (CFVIII), hyaluronic acid (HA), procollagen type III (PC-III), type IV collagen (C-IV), laminin (LN), etc. following the standard procedures.

\section{Biomedical measurement}

The test sera firstly were defrosted rapidly to room temperature before use. Then, the samples were tested by ELISA using secretory-excretory antigen (SEA) derived from eggs of $S$. japonicum. Meanwhile, the following control sera were used on each plate: a reference positive sera (serum from a patient with microscopically confirmed S. japonicum infection), and a tropical negative serum (serum from an individual with no evidence of Schistosoma infection). D-dimer was assayed by immunoturbidimetric method (Siemens, no. 412056). The liver function tests, including ALB, GLB, TT, and CF-VIII, were measured by automated biochemistry analyzer (Roche, Basel, Swiss). Additional hepatic fibrosis indices, including HA, PC- 
III, C-IV, and LN, were measured by commercially available radioimmunoassay (RIA kits; Shanghai Navy Medical Institution, Shanghai, China). To control the confounding effects of hepatitis B virus (HBV) infection, determinations of HBsAg and anti-HBc were made by automatic light detector and test reagents (Abbott, North Chicago, Illinois, USA). Abdominal ultrasonography was employed to identify the activity of ascites and to observe the morphology of the liver and portal system. Accordingly, hepatomegaly was defined when the thickness of the left lobe was more than $70 \mathrm{~mm}$ or the major diameter of the right lobe was over $140 \mathrm{~mm}$; portal hypertension was defined when the width of the inner portal vein was no less than $12 \mathrm{~mm}$.

\section{Diagnostic criteria of schistosomiasis}

According to the criteria made by the Ministry of Health of $\mathrm{P}$. R. China, schistosomiasis is defined as (1) long-term or repeating water contact, or (2) finding eggs or miracidia by stool examination, or (3) finding eggs by transrectal biopsy, or (4) serum immunological tests positive. Advanced schistosomiasis is defined as (1) schistosomiasis patients suffering from portal hypertension of schistosomal liver fibrosis or colon granuloma, or (2) schistosomiasis patients with hepatomegaly (mainly in the left lobe).

\section{Data analysis}

Continuous variables were described by means and standard deviation or median with quartiles and were further compared between groups with appropriate statistical methods based on their distributions. Categorical variables were described with absolute values and percentages and further tested by a series of $\chi^{2}$ tests to examine the associations with ascites in the year 2014.

Defining occurrence of ascites as the state variable, we employed Receiver Operating Curve (ROC) to analyze the predictive value of each significantly different factor between groups. The greatest sum of specificity and sensitivity in the coordinator of the curve then determined the D-dimer's cutoff value for ascites, which further divided D-dimer levels into 2 groups. Then, we combined all of those categorical variables in a predictive logistic regression model whose dependent variable was the occurrence of ascites in 2014. Thus, predicted probability values were used as the prediction variable in a final ROC analysis of ascites. The Area under the Curve (AUC) was used to judge the performance of this predictive model. The probability of ascites occurrence in 2014 in different combination of risk factors was finally estimated using the final prediction model.

All $P$-values were 2-sided, and type I error was set as 0.05 . All statistical analyses were conducted with SPSS version 18.0 (SPSS Inc. Chicago, Illinois, USA).

\section{RESULTS}

\section{Characteristics of advanced patients and liver functions}

In a total of 291 patients we recruited in 2013, ascites occurred in 44 of them in 2014. Except for 3 cases (6.8\%) with ascites in the liver area, all other patients' ascites were confined in the kidney area. The median area of the liquid grey area detected by ultrasound was $20 \mathrm{~mm}^{2}$ with a range of 8 to $61 \mathrm{~mm}^{2}$. As shown in Table 1, patients' D-dimer levels in 2013 were significantly higher in patients who developed to ascites in 2014 $(0.71 \pm 2.44 \mu \mathrm{g} / \mathrm{L})$, compared to the control group $(0.48 \pm 2.12$ $\mu \mathrm{g} / \mathrm{L}$ ). Additionally, the case group had more proportions of males and level 2 hepatic fibrosis in 2013 than in the control group. We also found significantly lower ALB while higher type IV collagen in the case group. Patients with obstinate ascites are also more likely developing to collateral circulation with splenomegaly and subsequent splenectomy (see an advanced case of schistosomiasis with ascites in the endemic area in 2014 in Fig. 1A and excised spleen in Fig. 1B). No significant differences in other liver function indicators, as well as age, occupation, HBV infection status, treatment during 2014, and portal hypertension were found between the case and control groups (Table 1).

\section{Predicted ascites occurrence with D-dimer and other factors as potential prognostic indicators}

Gender, hepatic fibrosis level, ALB, type IV collagen, and Ddimer, which were significantly different between cases and control groups, were used as independent variables to predict the occurrence of ascites in 2014. As shown in Table 2, the AUC of D-dimer was 0.64 with a $95 \%$ confidence interval of 0.54 to 0.73 . The AUC of other indicators were also significantly greater than 0.5 .

\section{Combined prediction model for ascites in 2014}

The logistic model which combined all the 5 significantly differentiated indicators of gender, hepatic fibrosis level, ALB, type IV collagen, and D-dimer was significant with R square as 
Table 1. Comparison of the general, infective, and hepatic characteristics in baseline (2013) between the cases of advanced schistosomiasis with/without ascites in 2014

\begin{tabular}{|c|c|c|c|}
\hline \multirow{2}{*}{ Baseline measurement } & \multicolumn{2}{|c|}{ Ascites occurrence in 2014} & \multirow{2}{*}{$P$-value } \\
\hline & Yes $(n=44)$ & No $(n=247)$ & \\
\hline Age (mean $\pm S D$, year) & $62.83 \pm 9.68$ & $61.80 \pm 9.71$ & 0.443 \\
\hline Gender & & & 0.026 \\
\hline Male & $33(75.0)$ & $141(57.1)$ & \\
\hline Female & $11(25.0)$ & $106(42.9)$ & \\
\hline Occupation & & & 0.483 \\
\hline Fisher & $27(61.4)$ & $163(66.0)$ & \\
\hline Farmer & $15(34.1)$ & 65 (26.3) & \\
\hline Others & $2(4.5)$ & $19(7.7)$ & \\
\hline HBV infection in 2013 & & & 0.996 \\
\hline Negative & $11(25.0)$ & $61(25.4)$ & \\
\hline Antibody & $26(59.1)$ & $142(59.2)$ & \\
\hline Positive & $7(15.9)$ & $37(15.4)$ & \\
\hline Hepatic fibrosis level in 2013 & & & 0.075 \\
\hline Level 1 & $4(9)$ & $36(15)$ & \\
\hline Level 2 & $13(30)$ & $104(42)$ & \\
\hline Level 3 & $27(61)$ & $105(43)$ & \\
\hline Portal hypertension in 2013 & & & 0.337 \\
\hline Yes & $10(22.7)$ & $41(16.7)$ & \\
\hline No & 34 (77.3) & 204 (83.3) & \\
\hline Treatment in 2014 & & & 0.107 \\
\hline Yes & $33(75.0)$ & $154(62.3)$ & \\
\hline No & $11(25.0)$ & $93(37.7)$ & \\
\hline D-dimer (mg/L) & $0.71 \pm 2.44$ & $0.48 \pm 2.12$ & 0.005 \\
\hline ELISA (SEA lgG, OD value) & $0.34(0.15-0.58)$ & $0.27(0.17-0.47)$ & 0.248 \\
\hline ALB (g/L) & $44.5(40.8-45.8)$ & $46.2(44.0-48.6)$ & $<0.001$ \\
\hline GLB (g/L) & $29.3(24.8-31.8)$ & 27.6 (24.7-30.3) & 0.101 \\
\hline$A / G$ & $1.5(1.4-1.8)$ & $1.7(1.5-1.9)$ & 0.006 \\
\hline $\mathrm{HA}(\mu \mathrm{g} / \mathrm{L})$ & $186.5(122.8-260.8)$ & $151.8(105.4-234.6)$ & 0.109 \\
\hline PC-III ( $\mu \mathrm{g} / \mathrm{L})$ & 83.5 (71.3-113.5) & $77.6(63.8-93.4)$ & 0.053 \\
\hline Type IV collagen ( $\mu \mathrm{g} / \mathrm{L})$ & $50.0(43.2-73.3)$ & $44.5(27.1-57.6)$ & 0.006 \\
\hline LN ( $\mu \mathrm{g} / \mathrm{L})$ & $124.7(108.7-152.3)$ & 125.9 (102.6-147.2) & 0.453 \\
\hline
\end{tabular}
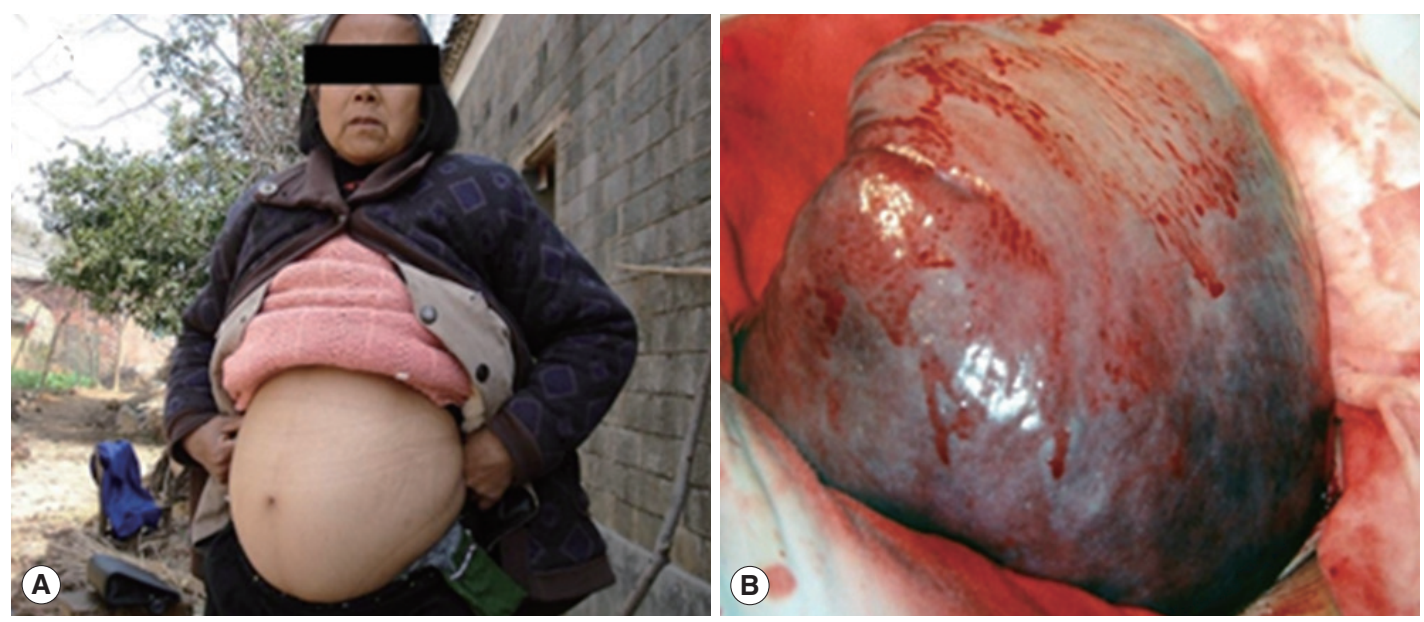

Fig. 1. (A) A patient of advanced schistosomiasis with ascites in the endemic area (2014, photo by one of the authors). (B) Excised spleen of a patient which developed to collateral circulation with splenomegaly (2009, photo by one of the authors). 
Table 2. The area under curve of each individual predictor for the occurrence of ascites in 2014

\begin{tabular}{|c|c|c|c|c|c|}
\hline \multirow{2}{*}{ Indicators } & \multirow{2}{*}{ Area } & \multirow{2}{*}{ Std. error } & \multirow{2}{*}{$P$-value } & \multicolumn{2}{|c|}{ Asymptotic 95\% confidence interval } \\
\hline & & & & Lower bound & Upper bound \\
\hline D-dimer & 0.635 & 0.050 & 0.004 & 0.537 & 0.733 \\
\hline Gender & 0.591 & 0.045 & 0.058 & 0.537 & 0.733 \\
\hline Hepatic fibrosis level & 0.595 & 0.046 & 0.045 & 0.505 & 0.685 \\
\hline ALB & 0.697 & 0.042 & $<0.001$ & 0.615 & 0.779 \\
\hline Type IV collagen & 0.641 & 0.046 & 0.006 & 0.550 & 0.730 \\
\hline
\end{tabular}

Table 3. The final inclusive prediction model of ascites occurrence in 2014

\begin{tabular}{|c|c|c|c|c|c|c|}
\hline & & Beta & Standardized error & Wald & $P$-value & OR (95\% Cl) \\
\hline Constant & & -2.516 & 0.444 & 32.060 & 0.000 & $0.081(\mathrm{NA})$ \\
\hline \multirow[t]{2}{*}{ Gender } & Female & Reference & - & - & - & - \\
\hline & Male & 0.942 & 0.450 & 4.376 & 0.036 & $2.57(1.06-6.20)$ \\
\hline \multirow[t]{2}{*}{ D-dimer } & $\leq 0.81 \mathrm{ng} / \mathrm{mL}$ & Reference & - & - & - & - \\
\hline & $>0.81 \mathrm{ng} / \mathrm{mL}$ & 0.82 & 0.399 & 4.219 & 0.040 & $2.27(1.04-4.96)$ \\
\hline Hepatic & Class 2 & Reference & - & - & - & - \\
\hline Fibrosis level & Class 3 & 0.29 & 0.394 & 0.540 & 0.462 & $1.34(0.62-2.90)$ \\
\hline \multirow[t]{3}{*}{ ALB } & 34-48 g/L (normal) & Reference & - & - & - & - \\
\hline & $<34$ g/L (lower) & 0.609 & 1.461 & 0.174 & 0.677 & $1.84(0.11-32.25)$ \\
\hline & $\geq 48$ g/L (higher) & -1.551 & 0.638 & 5.906 & 0.015 & $0.21(0.06-0.74)$ \\
\hline \multirow[t]{2}{*}{ Type IV Collagen } & $<75 \mu \mathrm{g} / \mathrm{L}$ & Reference & - & - & - & - \\
\hline & $\geq 75 \mu \mathrm{g} / \mathrm{L}$ & 0.879 & 0.512 & 2.950 & 0.086 & $2.41(0.88-6.57)$ \\
\hline
\end{tabular}

Table 4. Probability of ascites occurrence in combinations of risk factors

\begin{tabular}{|c|c|c|c|c|c|c|}
\hline $\begin{array}{l}\text { Combinations of } \\
\text { risk factors }\end{array}$ & Gender & $\begin{array}{c}\text { Higher level of } \\
\text { D-dimer }\end{array}$ & $\begin{array}{l}\text { Level } 3 \text { hepatic } \\
\text { fibrosis }\end{array}$ & Higher ALB & Higher TIC & Probability (\%) \\
\hline 1 & Male & Yes & Yes & Yes & Yes & 73.5 \\
\hline 2 & Male & Yes & No & Yes & Yes & 67.5 \\
\hline 3 & Male & Yes & Yes & No & Yes & 60.2 \\
\hline 4 & Male & No & Yes & Yes & Yes & 55.0 \\
\hline 5 & Male & Yes & Yes & Yes & No & 53.6 \\
\hline 6 & Male & Yes & No & No & Yes & 53.1 \\
\hline 7 & Female & Yes & Yes & Yes & Yes & 52.0 \\
\hline
\end{tabular}

0.208. As shown in Table 3, hepatic fibrosis level fell out of a significant predictor $(\mathrm{OR}=1.34,95 \% \mathrm{CI}$ : 0.62-2.90) when included with other factors into the final inclusive model. Nevertheless, the performance of this model was better than any individual predictors with the AUC as 0.76 with a 95\% confidence interval of 0.68-0.89. In addition, the prediction model can be translated into the following equation for the probability of the occurrence of ascites:

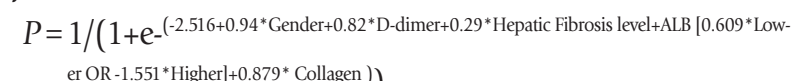
er OR $-1.551^{*}$ Higher $+0.879^{*}$ Collagen $\left.)\right)$.

This equation would be easily used for clinics since all of the predictors were binary. Practitioners can input the value as 0 or 1 into the equation then get the probability of the occurrence of ascites.

Finally, the probability of incident ascites was found to be as largest as $73.5 \%$ when an advanced case is a male, with higher level D-dimer, ALB, C-III, and level 3 hepatic fibrosis. Followed by is a probability of $67.5 \%$ when a patient has all these positive risk factors except for level 3 hepatic fibrosis. The probability will decrease to $60.2 \%, 55.0 \%$, and 53.6 when patients have all risk factors except for 1 symptom of higher ALB, higher D-dimer, or higher TIC, respectively. We listed 7 combinations of the risk factors with the probability in Table 4 for clinic practitioners to refer when these easy-achievable test results are available. 


\section{DISCUSSION}

In this nested case-control study, we observed 44 cases of incident ascites in 291 patients with advanced schistosomiasis japonica and found higher levels of plasma D-dimer as an independent risk factor. D-dimer may predict the prospective occurrence of ascites in combination with other traditional clinical risk factors of gender as male, grade III hepatic fibrosis, high level of ALB, and high level of type IV Collagen.

Ascites is a dominant symptom in advanced schistosomiasis at the current endemic control stage in China. In our 1-year prospective study, 44 out of 291 patients revealed ascites at the end of the follow-up period with an incidence rate of $15.1 \%$. Given the high proportion around $65 \%$ of ascites subtype in advanced cases across the whole endemics [22,23], the incident rate in the present study was even lower than estimated in the transmission-active areas. Ascites was regarded as the trend symptom in advanced schistosomiasis based on an analyses of historical medical records of all patients of advanced schistosomiasis from 2004 to 2013 in Jinmen county, Hubei province [24]. We found males as a risk factor of ascites, which is consistent with previous studies conducted in the same regions but inconsistent with studies conducted in Yunnan where women are the major labor [25]. However, we did not find significant differences in the distributions of occupations across cases and control groups. Occupation was previously regarded as an indicator of exposure to Schistosoma-infected water and occupations with more contacts, such that fishers and farmers of paddy fields are in higher risk [26]. However, with the popularity of machine-farming practice and inhibition of disease transmission, people in those traditional high-risk jobs may be well protected now. Hepatitis B virus infection was previously found to be interacting with schistosomiasis in the liver damaging but we did not find a significance association of HBV infection status with incident ascites in the present study [27]. However, we did find there still remaining $15 \%$ patients with active HBV infection while another half (59\%) were immunized. This finding indicates a remaining need of HBV treatment in patients with co-infection of both $S$. japonicum and HBV.

With abdomen ultrasound, we found patients having grade III hepatic fibrosis were more tend to have ascites than those without. Even though the association was marginally significant $(P=0.075)$, the trend was still meaningful and provided evidence for the need of treatment to fibrosis in advanced $S$. japonicum. Traditional treatment to $S$. japonicum was mostly targeting at antigen, especially with an extensive usage of praziquantel when apparent symptoms are absent. However, more and more pieces of evidence are indicating that liver damage is constantly advancing along with fibrogenesis caused by Schistosoma eggs. Yin et al. [28] investigated 75 newly diagnosed advanced $S$. japonicum in transmission-blocked areas and found that $82.7 \%$ patients had grade III hepatic fibrosis. They suggested the liver of patients with infection history kept being insulted by the eggs' residuals, such as proteins and lipopolysaccharide, albeit the larva in the eggs were killed by antigen treatment and patients were free from reinfection. Similarly, we also found worse liver function in patients with incident of ascites that their ALB was significantly lower than those without, but lack significant differences in SEA IgG levels. We also found an indicator of hepatic fibrosis, type IV collagen level, was significantly higher in the case group. All these findings indicated the association of ascites with enduring liver damage and fibrogenesis.

The mechanisms of ascites in advanced schistosomiasis have been widely studied, including physical obstruction caused by eggs and periovular granulomas, liver fibrosis, and portal vein hypertension, along with decreased plasma colloid osmotic pressure [1]. However, the mechanism of liver fibrosis is relatively less studied. In the current study, we found increased levels of D-dimers in patients with incident ascites compared with those without. D-dimer is the primary product when cross-lined fibrin is degraded by plasmin-induced fibrinolytic activity, which is correlated with intravascular levels of fibrin turnover [17]. Thus, D-dimer was regarded as a marker of fibrin turnover, which reflects impairment in coagulation and fibrinolysis pathways. This abnormal function may lead to hepatic fibrosis, as we observed in those patients with incident ascites. Infection of Schistosoma may lead to blood vessel injury and subsequent activate systems involving inflammation, coagulation, and fibrinolysis [29,30]. Additionally, the liver damage will also confine the synthesis of anti-coagulation proteins and thus further aggravate the imbalance to the tendency of thrombosis [29] and increase the risk of liver fibrosis. All of these malfunctions eventually lead to the occurrence of ascites.

Given the critical role of D-dimer in the aforementioned mechanism of ascites and hepatic fibrosis, we combined D-dimer with traditional clinical easy-achievable indicators to predict the incidence of ascites in patients with advanced schistosomiasis japonica. The combination is more capable in pre- 
dicting future incidence of ascites than any other indicators on their own. Conventionally a prediction with an area under the ROC curve (AUROC) greater than 0.7 would be regarded as usable in clinics [31]. Thus, the AUROC of the combination of D-dimer with other indicators as 0.76 with a 95\% interval of 0.68-0.89 demonstrated its usefulness for a clinical value. As such, we calculated the 7 highest possibilities of future ascites for clinical practitioners to make better decisions when providing treatments to patients with advanced schistosomiasis japonica. Clinical practitioners could use Table 4 as a reference to check the possibility of future ascites incidence risk in patients who may not have any symptoms presently. However, our results are still limited with a small number of samples and relatively short observation time. So, further studies with more representative samples and longer study duration would guarantee to verify the prediction model.

Our study has its own limitations. First, we obtained patients' sera only once in the year 2013. Although no patients had the symptom of ascites in 2013 when sera were collected, more measurements of D-dimer during the follow-up may provide more accuracy in the prediction model of ascites. Second, our study sample was small in number and follow-up duration was short, which may limit the power to detect more meaningful indicators of incident ascites. Third, we included a number of patients (15\%) co-infected with HBV. Patients of advanced schistosomiasis associated with HBV infection usually show persistent viremia and exhibit chronic active hepatitis, which can progress to cirrhosis $[13,14]$. Even though we set HBV infection status as a confounding factor throughout the analyses process, the proportion of ascites contributed by HBV cannot be thoroughly eliminated.

In conclusion, we suggest that plasma D-dimer is a reliable predictor for incident ascites in advanced schistosomiasis japonica in combination with other clinical easy-achievable indicators. However, further studies with more representative samples and longer study duration would guarantee to verify the prediction model.

\section{ACKNOWLEDGMENTS}

We would like to thank the clinical team in the local hospital for their great assistance in data collection. This study was supported by the China Postdoctoral Science Foundation (grant no. 2016M591605), National Research and Development Plan of China (no. 2016YFC1200500) and Jiangxi Pro- vincial Key Technology Support Project (grant no. 2011 wbbg70001).

\section{CONFLICT OF INTEREST}

We have no conflict of interest related to this work.

\section{REFERENCES}

1. Colley DG, Bustinduy AL, Secor WE, King CH. Human schistosomiasis. Lancet 2014; 383: 2253-2264.

2. Gray DJ, McManus DP, Li Y, Williams GM, Bergquist R, Ross AG. Schistosomiasis elimination: lessons from the past guide the future. Lancet Infect Dis 2010; 10: 733-736.

3. Chen MG. Assessment of morbidity due to Schistosoma japonicum infection in China. Infect Dis Poverty 2014; 3: 6.

4. Yang Y, Zhou YB, Song XX, Li SZ, Zhong B, Wang TP, Bergquist R, Zhou XN, Jiang QW. Integrated control strategy of schistosomiasis in China: Projects involving agriculture, water conservancy, forestry, sanitation and environmental modification. Adv Parasitol 2016; 92: 237-268.

5. Lei ZL, Zhang LJ, Xu ZM, Dang H, Xu J, Lv S, Cao CL, Li SZ, Zhou XN. Endemic status of schistosomiasis in People's Republic of China in 2014. Zhongguo Xue Xi Chong Bing Fang Zhi Za Zhi 2015; 27: 563-569.

6. Zhao GM, Zhao Q, Jiang QW, Chen XY, Wang LY, Yuan HC. Surveillance for schistosomiasis japonica in China from 2000 to 2003. Acta Trop 2005; 96: 288-295.

7. Li SZ, Luz A, Wang XH, Xu LL, Wang Q, Qian YJ, Wu XH, Guo JG, Xia G, Wang LY, Zhou XN. Schistosomiasis in China: acute infections during 2005-2008. Chin Med J (Engl) 2009; 122: 1009-1014.

8. Zhou XN, Guo JG, Wu XH, Jiang QW, Zheng J, Dang H, Wang $\mathrm{XH}, \mathrm{Xu}$ J, Zhu HQ, Wu GL, Li YS, Xu XJ, Chen HG, Wang TP, Zhu YC, Qiu DC, Dong XQ, Zhao GM, Zhang SJ, Zhao NQ, Xia G, Wang LY, Zhang SQ, Lin DD, Chen MG, Hao Y. Epidemiology of schistosomiasis in the People's Republic of China, 2004. Emerg Infect Dis 2007; 13: 1470-1476.

9. Olds GR, Olveda R, Wu G, Wiest P, McGarvey S, Aligui G, Zhang S, Ramirez B, Daniel B, Peters P, Romulo R, Fevidal P, Tiu W, Yuan J, Domingo E, Blas B. Immunity and morbidity in schistosomiasis japonicum infection. Am J Trop Med Hyg 1996; 55: 121-126.

10. Zhang JF, Wen LY, Zhu MD, Yan XL, Chen W, Li L, Lin LJ, Yu LL. Recent investigation and treatment of 1060 advanced schistosomiasis cases in Zhejiang Province. Zhongguo ji sheng chong xue yu ji sheng chong bing za zhi 2010; 28: 214-217.

11. Yu LQ. Clinical analysis on 121 cases of Advanced schistosomiasis in old people. J Huaihai Med 2014; 32: 583-584.

12. Jia TW, Utzinger J, Deng Y, Yang K, Li YY, Zhu JH, King CH, Zhou XN. Quantifying quality of life and disability of patients 
with advanced schistosomiasis japonica. PLoS Negl Trop Dis 2011; 5: e966.

13. Perrier A, Desmarais S, Miron MJ, de Moerloose P, Lepage R, Slosman D, Didier D, Unger PF, Patenaude JV, Bounameaux H. Non-invasive diagnosis of venous thromboembolism in outpatients. Lancet 1999; 353: 190-195.

14. Goldhaber SZ, Simons GR, Elliott CG, Haire WD, Toltzis R, Blacklow SC, Doolittle MH, Weinberg DS. Quantitative plasma D-dimer levels among patients undergoing pulmonary angiography for suspected pulmonary embolism. JAMA 1993; 270: 2819-2822.

15. Spadaro A, Tortorella V, Morace C, Fortiguerra A, Composto P, Bonfiglio C, Alibrandi A, Luigiano C, De Caro G, Ajello A, Ferrau $\mathrm{O}$, Freni MA. High circulating D-dimers are associated with ascites and hepatocellular carcinoma in liver cirrhosis. World J Gastroenterol 2008; 14: 1549-1552.

16. Goldhaber SZ, Vaughan DE, Tumeh SS, Loscalzo J. Utility of cross-linked fibrin degradation products in the diagnosis of pulmonary embolism. Am Heart J 1988; 116: 505-508.

17. Ge LP, Li J, Bao QL, Chen P, Jiang Q, Zhu LR. Prognostic and predictive value of plasma D-dimer in advanced non-small cell lung cancer patients undergoing first-line chemotherapy. Clin Transl Oncol 2015; 17: 57-64.

18. Primignani M1, Dell'Era A, Bucciarelli P, Bottasso B, Bajetta MT, de Franchis R, Cattaneo M. High-D-dimer plasma levels predict poor outcome in esophageal variceal bleeding. Dig Liver Dis 2008; 40: 874-881.

19. Andrade ZA. Schistosomiasis and liver fibrosis. Parasite Immunol 2009; 31: 656-663.

20. MOH. Manual of Schistosomiasis prevention and control. Third Edition ed. Shanghai: Shanghai Science and Technology Press; 2000.

21. Wu E. Medical Imageology. 6th ed. Wu E, editor: People's Medical Publishing House; 2007.

22. Liu ZC, Xiao SY, Zhou J, Yu XL, Hu BJ, Zhu JH, Li YS. Analysis of epidemiological characteristics of advanced schistosomiasis in
Hunan Province. Zhongguo Xue Xi Chong Bing Fang Zhi Za Zhi 2014; 26: 148-152.

23. Zhang SQ GF-H, He JX, Zhang GH, Wang H, Yang WP, Wang TP. Endemic status of schistosomiasis in Anhui Province in 2014. J Trop Dis \& Parasitol 2015; 13: 131-134,174.

24. Ding ZJ. Advanced schistosomiasis prevalence in Jingmen City from 2004 to 2013. Zhongguo xue xi chong bing fang zhi za zhi 2014; 26: 559-560.

25. Zhang XY ZZ T, Dong Y, Zhang Y, Feng XG, Shen MF, Dong XQ. Distribution and characteristics of advanced schistosomiasis in Yunnan Province. J Trop Dis \& Parasitol 2010; 8: 204-206.

26. Ross AG, Yuesheng L, Sleigh AS, Yi L, Williams GM, Wu WZ, Xinsong L, Yongkang H, McManus DP. Epidemiologic features of Schistosoma japonicum among fishermen and other occupational groups in the Dongting Lake region (Hunan Province) of China. Am J Trop Med Hyg 1997; 57: 302-308.

27. Domingo EO, Lingao AL, Tiu E, Lao JY, Olveda RM. HBV exposure and HBsAg positivity rates in schistosomiasis japonica: study in a Philippine community endemic for both infections. Southeast Asian J Trop Med Public Health 1983; 14: 456-462.

28. Yin AH, Hua HY, Sun GX, Xu MG, Zhou WE, Zhu WC, Feng JY, You L, Tang F, Liang YS. Survey on newly-developed advanced schistosomiasis patients in schistosomiasis transmission-interrupted areas. Zhongguo xue xi chong bing fang zhi za zhi 2013; 25: 477-480, 484.

29. Antoniou D, Pavlakou G, Stathopoulos GP, Karydis I, Chondrou E, Papageorgiou C, Dariotaki F, Chaimala D, Veslemes M. Predictive value of $\mathrm{D}$-dimer plasma levels in response and progressive disease in patients with lung cancer. Lung Cancer 2006; 53: 205-210.

30. Zhang DL, Hao JY, Yang N. Value of D-dimer and protein S for diagnosis of portal vein thrombosis in patients with liver cirrhosis. J Int Med Res 2013; 41: 664-667.

31. Alemayehu D, Zou KH. Applications of ROC analysis in medical research: recent developments and future directions. Acad Radiol 2012; 19: 1457-1464. 